\title{
Compressive Online Robust Principal Component Analysis with Multiple Prior
} Information

\author{
Van Luong, Huynh; Deligiannis, Nikos; Seiler, Jürgen; Kaup, Andre; Forchhammer, Søren
}

Publication date:

2017

Document Version

Publisher's PDF, also known as Version of record

Link back to DTU Orbit

Citation (APA):

Van Luong, H., Deligiannis, N., Seiler, J., Kaup, A., \& Forchhammer, S. (2017). Compressive Online Robust Principal Component Analysis with Multiple Prior Information. Poster session presented at 5th IEEE Global Conference on Signal and Information Processing, Montreal, Canada.

\section{General rights}

Copyright and moral rights for the publications made accessible in the public portal are retained by the authors and/or other copyright owners and it is a condition of accessing publications that users recognise and abide by the legal requirements associated with these rights.

- Users may download and print one copy of any publication from the public portal for the purpose of private study or research.

- You may not further distribute the material or use it for any profit-making activity or commercial gain

- You may freely distribute the URL identifying the publication in the public portal 
COMPRESSIVE ONLINE ROBUST PRINCIPAL COMPONENT ANALYSIS

\section{WITH MULTIPLE PRIOR INFORMATION}

Huynh Van Luong ${ }^{1}$, Nikos Deligiannis², Jürgen Seiler ${ }^{1}$, André Kaup ${ }^{1}$, and Søren Forchhammer ${ }^{3}$

Multimedia Communications and Signal Processing, University of Erlangen-Nuremberg, 91058 Erlangen, Germany

2Vrije Universiteit Brussel, 1050 Brussels, Belgium and imec, Kapeldreef 75, B-3001 Leuven, Belgium

${ }^{3}$ DTU Fotonik, Technical University of Denmark, 2800 Lyngby, Denmark

\section{Motivation}

- Applications: Computer vision, web data analysis, anomaly detection, and data visualization, etc.

- Robust Principal Component Analysis (RPCA): Batch-based, - Recompos sparse $(\boldsymbol{S})$, e g, all frames in a video, high computational and memory requirements $\min _{\boldsymbol{L}, \boldsymbol{S}}\|\boldsymbol{L}\|_{*}+\lambda\|\boldsymbol{S}\|_{1}$ subject to $\boldsymbol{M}=\boldsymbol{L}+\boldsymbol{S}$

Challenges

- Online method processing a sequence of signals per time

instance from a small set of measurements $y_{t}=\boldsymbol{\Phi}\left(x_{t}+\boldsymbol{v}_{t}\right)$

$\boldsymbol{M}_{t}=\boldsymbol{L}_{t}+\boldsymbol{S}_{t}$ into $\boldsymbol{S}_{t}=\left[\begin{array}{lllll}\boldsymbol{x}_{1} & \boldsymbol{x}_{1} & \ldots & \boldsymbol{x}_{t}\end{array}\right]$ and $\boldsymbol{L}_{t}=\left[\begin{array}{llll}\boldsymbol{v}_{1} & \boldsymbol{v}_{2} & \ldots & \boldsymbol{v}_{t}\end{array}\right]$

$>$ Minimization at time instance

$\min _{\boldsymbol{x}_{t}, \boldsymbol{v}_{t}}\left\|\left[\boldsymbol{L}_{t-1} \boldsymbol{v}_{t}\right]\right\|_{*}+\lambda\left\|\boldsymbol{x}_{t}\right\|_{1}$ subject to $\boldsymbol{y}_{t}=\boldsymbol{\Phi}\left(\boldsymbol{x}_{t}+\boldsymbol{v}_{t}\right)$

where $L_{t-1}=\left[\begin{array}{lll}v_{1} & v_{2} & v_{t-1}\end{array}\right] S_{t-1}=\left[x_{1} x_{1} \ldots x_{t-1}\right], \Phi$ are given

\section{Compressive Online RPCA (CORPCA) With Multiple Prior Information}

\begin{tabular}{|c|c|c|}
\hline 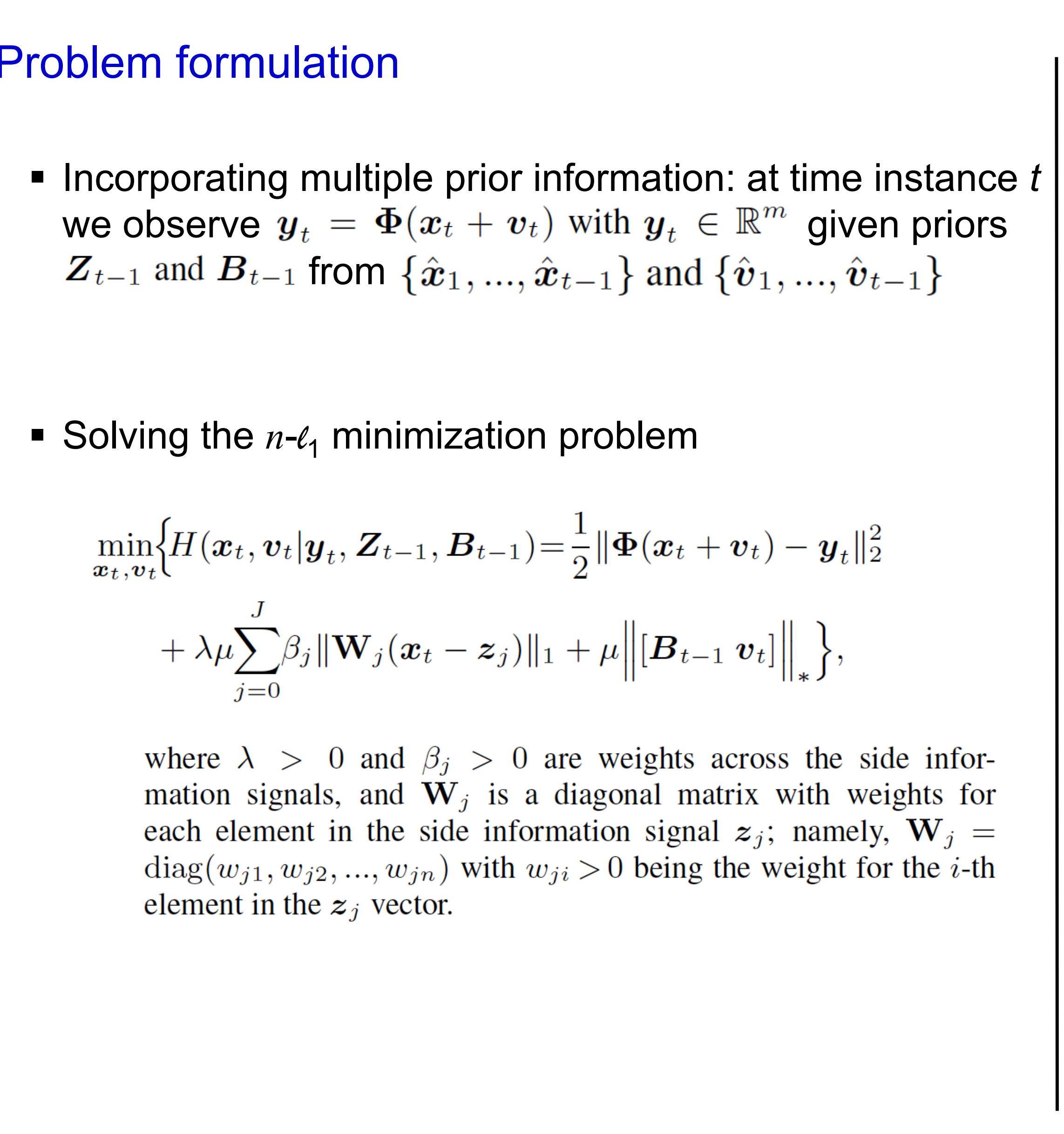 & $\begin{array}{l}\text { The CORPCA algorithm } \\
\text { - Solving } n-\ell_{1} \text { minimization via the soft thresholding } \\
\text { operator and the single value thresholding operator, at } \\
\text { iteration } k+1 \\
\quad \boldsymbol{v}_{t}^{(k+1)}=\underset{v_{t}}{\arg \min }\left\{\mu h\left(\boldsymbol{v}_{t}\right)+\left\|\boldsymbol{v}_{t}-\left(\boldsymbol{v}_{t}^{(k)}-\frac{1}{2} \nabla_{v_{t}} f\left(\boldsymbol{v}_{t}^{(k)}, \boldsymbol{x}_{t}^{(k)}\right)\right)\right\|_{2}^{2}\right\} \\
\quad \boldsymbol{x}_{t}^{(k+1)}=\underset{\boldsymbol{x}_{t}}{\arg \min }\left\{\mu g\left(\boldsymbol{x}_{t}\right)+\left\|\boldsymbol{x}_{t}-\left(\boldsymbol{x}_{t}^{(k)}-\frac{1}{2} \nabla_{\boldsymbol{x}_{t}} f\left(\boldsymbol{v}_{t}^{(k)}, \boldsymbol{x}_{t}^{(k)}\right)\right)\right\|_{2}^{2}\right\} \\
\quad \text { where } f\left(\boldsymbol{v}_{t}, \boldsymbol{x}_{t}\right)=(1 / 2)\left\|\boldsymbol{\Phi}\left(\boldsymbol{x}_{t}+\boldsymbol{v}_{t}\right)-\boldsymbol{y}_{t}\right\|_{2}^{2} \\
\quad g\left(\boldsymbol{x}_{t}\right)=\lambda \sum_{j=0}^{J} \beta_{j}\left\|\mathbf{W}_{j}\left(\boldsymbol{x}_{t}-\boldsymbol{z}_{j}\right)\right\|_{1} \text {, and } h\left(\boldsymbol{v}_{t}\right)=\left\|\left[\boldsymbol{B}_{t-1} \boldsymbol{v}_{t}\right]\right\|_{*} \\
\text { - Updating weights } \beta_{j} \text { and } \mathbf{W}_{j} \\
\text { - After solving for time instance } t: \text { Prior updates } \\
\quad \boldsymbol{Z}_{t}:=\left\{\boldsymbol{z}_{j}=\boldsymbol{x}_{t-J+j}\right\}_{j=1}^{J} \\
\quad \boldsymbol{B}_{t}=\boldsymbol{U}_{t}(:, 1: d) \boldsymbol{\Gamma}_{\frac{\mu_{k}}{2} g_{1}}\left(\boldsymbol{\Sigma}_{t}\right)(1: d, 1: d) \boldsymbol{V}_{t}(:, 1: d)^{\mathrm{T}}\end{array}$ & 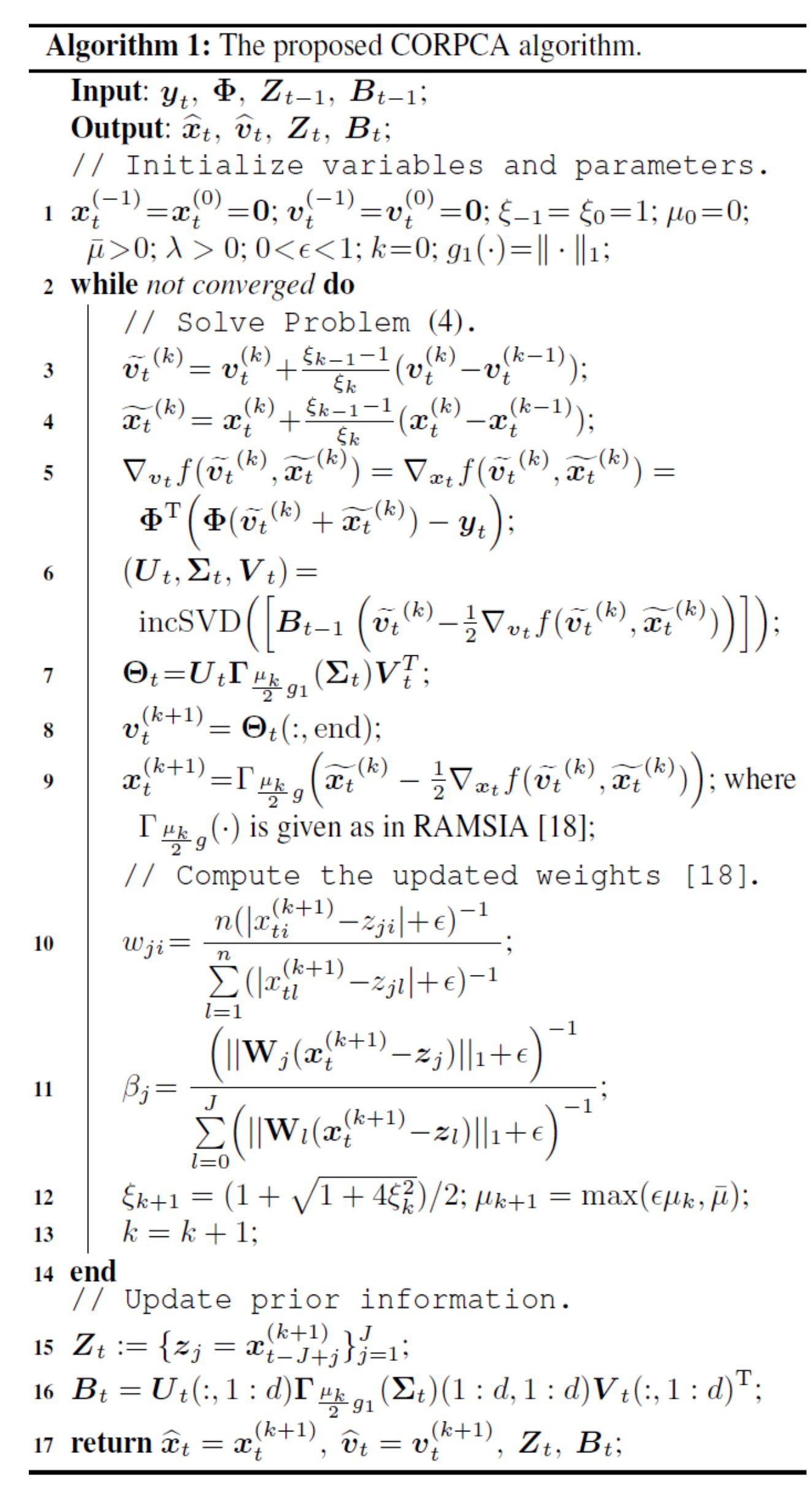 \\
\hline
\end{tabular}

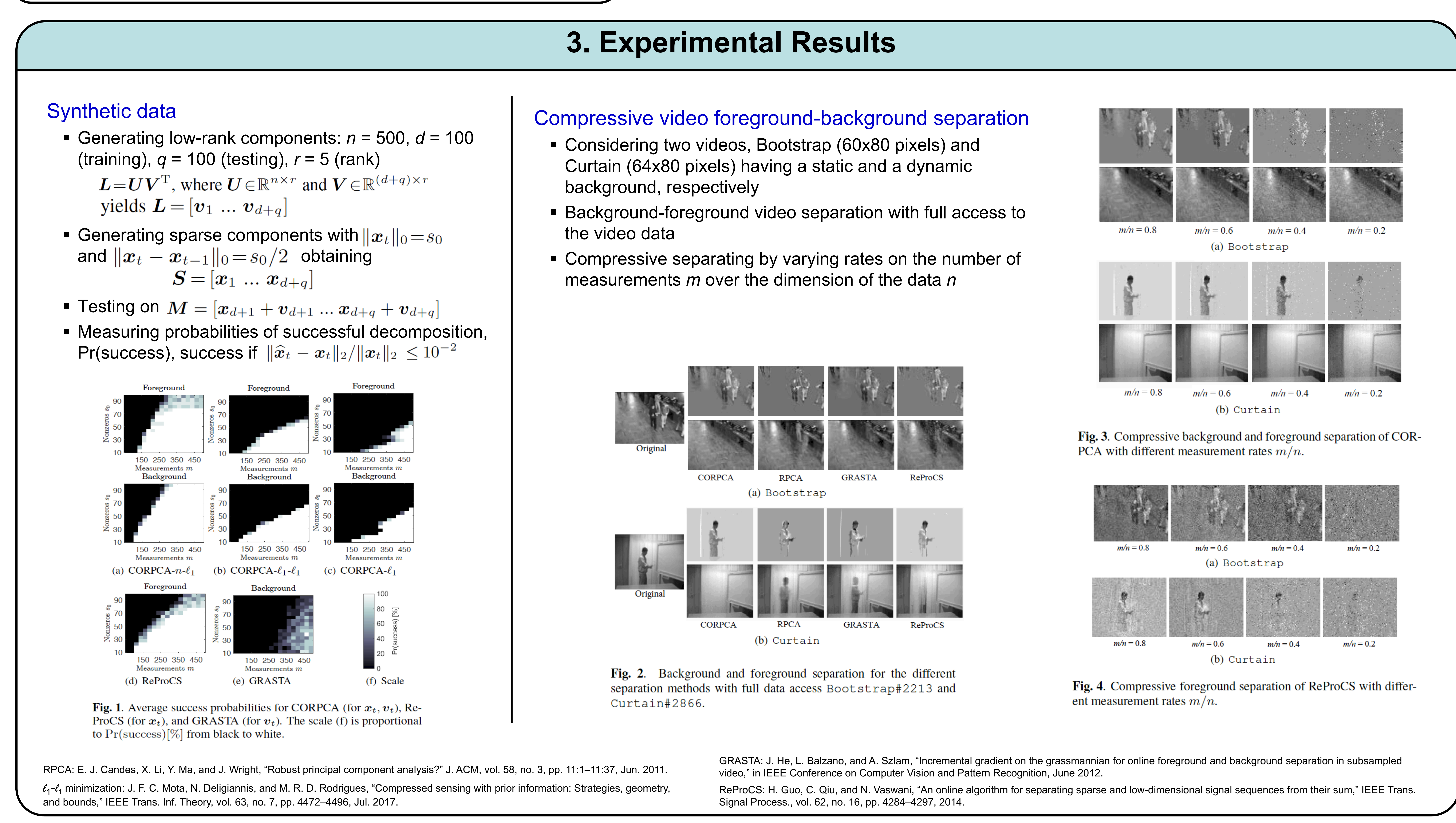

\begin{tabular}{|l|}
\hline \multicolumn{1}{|c|}{ 4. Summary } \\
\hline Solution for an $n-\ell_{1}$ minimization \\
- Incorporating efficiently multiple prior information \\
- Updating iteratively weights \\
The proposed COPRCA algorithm \\
- Processing a data vector per time instance using \\
compressive measurements \\
- Solving the $n-\ell_{1}$ minimization and updating priors for the \\
next instance \\
Evaluation of COPRCA on synthetic data and actual \\
video data \\
- Outperforming classical compressive sensing (CS) $\left(\ell_{1}\right.$ \\
minimization) and CS with single prior information $\left(\ell_{1}-\ell_{1}\right.$ \\
minimization) \\
- The superior performance improvement compared to the \\
existing methods
\end{tabular}

